\title{
Nano-interconnection for microelectronics and polymers with benzo-triazole
}

\author{
Yeonjoon Park ${ }^{1}$, Sang H. Choi ${ }^{2}$, Hyunpil Noh ${ }^{3}$, and Young Kuk ${ }^{4}$ \\ ${ }^{1}$ Science and Technology Corp., Hampton, VA \\ ${ }^{2}$ NASA Langley Research Center, Hampton, VA \\ ${ }^{3}$ Samsung Electronics Co. Ltd., Seoul, Korea \\ ${ }^{4}$ Dept. of Physics, Seoul National University, Seoul, Korea
}

\begin{abstract}
Benzo-Triazole (BTA) is considered as an important bridging material that can connect an organic polymer to the metal electrode on silicon wafers as a part of the microelectronics fabrication technology. We report a detailed process of surface induced 3-D polymerization of BTA on the $\mathrm{Cu}$ electrode material which was measured with the Ultraviolet Photoemission Spectroscopy (UPS), X-ray Photoemission Spectroscopy (XPS), and Scanning Tunneling Microscope (STM). The electric utilization of shield and chain polymerization of BTA on Cu surface is contemplated in this study.
\end{abstract}

\section{Introduction}

It is an exciting and important challenge to fabricate an IC chip that can be connected to bio-materials, proteins, tissues, or neurons. In order to achieve this goal, the current nano-electronics requires certain bridge-materials that can connect the metal electrodes to the organic polymers. For example, a small fiber-fragment fails to connect two Au electrodes in Figure 1-(a). If there existed a certain bridge-material that can grab the metal electrode and fiber-fragment at the same time, current-voltage (I-V) characteristics of the fiber could be obtained in this micro circuit. As another example, Figure 1-(b) (d) show the manipulation of bio-protein, Ferritin with Atomic Force Microscope (AFM) tip. Ferritin is an important protein that stores the iron ions inside the hemoglobin of the blood cell. ${ }^{\mathrm{i}}$ ii Randomly distributed ferritin proteins were brought together to build a compact nano structure. The entire AFM manipulation took place inside a junction area of metal electrodes on a micro-chip, as shown in Figure 1-(e), (f), and (g). However, the final interconnections from the nearby metal electrodes to the arranged ferritin proteins are still missing. Therefore it is important to develop good materials that can act as a bridge between metal electrodes and bio proteins, organic polymers, and nano structures.

The common metal electrodes of Integrated Circuit (IC) chips are made with aluminum (Al), tungsten (W), gold (Au) and copper $(\mathrm{Cu}){ }^{\text {iii }}$ Among these metals, $\mathrm{Cu}$ has extremely large diffusion-coefficient ${ }^{\text {iv }}$ in silicon wafer and it often destroys many transistors. Therefore, $\mathrm{Cu}$ was accepted as an electrode material in the silicon technology, only after the diffusion problems were solved with Damascene process developed by IBM. ${ }^{\mathrm{iii}, \mathrm{v}}$

Benzotriazole (BTA) is an interesting molecule that is known to polymerize on $\mathrm{Cu}$ surface and form a protective layer against oxidation and etching of $\mathrm{Cu}$ electrodes and yet to be very conductive in the thin film thickness. Therefore, we report the detailed polymerization process of $\mathrm{BTA}^{\mathrm{vi}}$ once more in this conference and suggest the utilization of BTA to interconnect external organic polymers to the metal electrodes by functionalization of BTA molecules.

\section{Experiment and Results}

BTA is an organic molecule with a hexagonal benzene ring and pentagonal nitrogen ring as shown in Figure 2. Various different surface polymerization models have been proposed by many groups as shown in Figure 2. Roberts and Rubim et al. ${ }^{\text {vii }}$ suggested a flat surface adsorption while Boshung Fang et al. ${ }^{\text {viii }}$ suggested a vertical polymerization with nitrogen-hydrogen bond. Zhi Xu et al. measured the tilted angle of symmetric axis of BTA to be $62^{\circ}$ with dichroic ratio measurement, in an effort to support the vertical polymerization model. ${ }^{\mathrm{x}}$ Walsh et al. ${ }^{\mathrm{x}}$ suggested a vertical adsorption model of BTA on $\mathrm{Cu}(100)$ surface from a polarization dependent NEXAFS study. However, no clear conclusion has been made among these numerous studies.

BTA is known to have complex molecular orbitals as shown in Table 1. Molecular orbital (MO) P1 P5 are $\pi-$ MOs with anti symmetric property upon the central plane and S1 S5 are $\sigma-\mathrm{MOs}$ with symmetric property upon the central 
plane. $\mathrm{N}+$ and $\mathrm{N}$ - states are the nitrogen induced states. Note that $\mathrm{P} 1$ and $\mathrm{P} 2$ are hybrid $\pi$-MOs while $\mathrm{P} 3, \mathrm{P} 4$ and $\mathrm{P} 5$ are global $\pi-\mathrm{MO}$.

\begin{tabular}{|l|l|l|l|l|l|}
\hline MO Name & $\begin{array}{l}\text { Ionization } \\
\text { Energy (eV) }\end{array}$ & $\begin{array}{l}\text { Orbital } \\
\text { Assignment }\end{array}$ & MO Name & $\begin{array}{l}\text { Ionization } \\
\text { Energy(eV) }\end{array}$ & $\begin{array}{l}\text { Orbital } \\
\text { Assignment }\end{array}$ \\
\hline P5 & 9.08 & $\pi$ & S5 & 13.30 & $\sigma$ \\
\hline P4 & 9.49 & $\pi$ & P1 & 15.02 & $\pi, \sigma, \sigma, \sigma$ \\
\hline N- & 10.05 & $\mathrm{~N}-$ & S4 & 16.93 & $\sigma$ \\
\hline P3 & 11.44 & $\pi$ & S3 & 17.67 & $\sigma$ \\
\hline N+ & 11.93 & $\mathrm{~N}+$ & S2 & 19.60 & $\sigma, \sigma$ \\
\hline P2 & 12.77 & $\pi, \sigma$ & S1 & 20.10 & $\sigma$ \\
\hline
\end{tabular}

Table 1-Molecular Orbitals of BTA ${ }^{\text {viii, xi }}$

In our experiment, BTA was evaporated in ultra high vacuum chamber to be deposited on $\mathrm{Cu}(110)$ single crystal. The $\mathrm{Cu}(110)$ single crystal was cleaned with repeated $\mathrm{Ar}$ sputtering and annealing at $550^{\circ} \mathrm{C}$. Before the deposition, a sharp (1x1) reconstruction was confirmed with Low Energy Electron Diffraction (LEED). Additional X-ray Photoemission Spectroscopy (XPS) was taken to confirm the cleanness of $\mathrm{Cu}$ surface. Using the LEED, UV source was aligned $10^{\circ}$ off from the surface tangential direction toward [002] orientation of $\mathrm{Cu}(110)$ crystal in order to detect surface sate at $\bar{Y}$ direction. The UV detector was aligned $20^{\circ}$ off from the surface normal direction. Unpolarized He(I) $21.2 \mathrm{eV}$ light was used as the UV source. After the above setup, Ultraviolet Photoemission Spectroscopy (UPS) data was collected as a function of the BTA deposition time into the UHV chamber. The deposition time was controlled with a gate valve. Since the vapor pressure of BTA is $8 \times 10^{-6}$ torr at $300 \mathrm{~K}$, the evaporation of BTA occurred even at room temperature. The base pressure of UPS chamber was $2.0 \times 10^{-10}$ torr and the pressure was raised by $4 \times 10^{-10}$ torr during the deposition.

Figure 3-(a) shows the UPS data from clean $\mathrm{Cu}$ surface to $80 \mathrm{~min}$. after of BTA deposit and Figure 3-(b) shows the data portion from $80 \mathrm{~min}$. to $230 \mathrm{~min}$. of BTA deposit. In the beginning $\mathrm{Cu} 3 \mathrm{~d}$ band peaks are dominant, but BTA peaks start to appear and become stronger as more deposition occurs after $80 \mathrm{~min}$. In Figure 3-(a), the most prominent peak is labeled as "Initial Growth Peak" which was identified as BTA's P3 molecular orbital. At 230min. of BTA deposit, all the peaks, S4, P1, S5, P2, N+ and P3, can be identified as shown in Figure 3-(b). Figure 4 shows the net peak intensity of $80 \mathrm{~min}$. despot and $230 \mathrm{~min}$. deposit without the $\mathrm{Cu}$ background signal. Interestingly in the initial deposit of $80 \mathrm{~min}$., P3 was the strongest but P3 becomes weaker after $80 \mathrm{~min}$. when it is compared with other peaks at a later stage of deposit.

Figure 5-(A) shows the change in relative intensity when each peak intensity at $230 \mathrm{~min}$. deposit is taken as $100 \%$. Obviously, the global $\pi$-molecular orbital P3 takes a different trend-[1] of intensity growth while all other peaks follow the common trend-[2]. To understand this awkward phenomenon, we proposed an ab-initio calculation model in which BTA molecule changes its orientation depending on the deposit thickness since this model can explain the UPS intensity change of P3 MO.

Generally, the intensity of detected electrons in photoemission spectroscopy is proportional to the differential crosssection, $\frac{d \sigma}{d \Omega}$ of the sample, BTA, such as;

$\frac{d \sigma}{d \Omega}=\frac{\alpha}{\pi} \frac{k}{\omega}|\langle f|\hat{A} \cdot \vec{p}| i\rangle|^{2} \cdot a_{0}^{2}$

where $\mathrm{k}$ and $\omega$ are wave-number and angular frequency of the light, $\hat{A}$ is the polarization direction and also the vector potential direction of the light.

With the dipole approximation, $\hat{A} \cdot\langle f|\vec{p}| i\rangle=i m \omega_{f i} \hat{A} \cdot\langle f|\vec{x}| i\rangle$

Therefore the intensity is determined by

$\hat{A} \cdot\langle f|\vec{p}| i\rangle=\oint\left\{(\hat{A} \cdot \vec{x}) \Psi_{f}^{*}(\vec{x}) \Psi_{i}(\vec{x})\right\} d^{3} \vec{x}$

The P3 global $\pi$-molecular orbital has anti-symmetric property as shown in Figure 5-(B)-(a). Now, a total of six different cases can be considered with the polarization directions and BTA's positions. Four of the six cases are shown 
in Figure 5-(B)-(b) to Figure 5-(B)-(e), that are consistent with our experiment. In UPS, the final state can be approximated with a parallel traveling electron wave-function in z-direction, $\propto e^{i k z}$. In the case of Near Edge X-ray Absorption Fine Structure (NEXAFS) or X-ray Absorption Near-Edge Structure (XANES), the final state is the unoccupied excited molecular orbital.

Case (1): BTA adsorbed flat on $\mathrm{Cu}$ and the light's polarization is parallel with surface, as shown in Figure 5-(B)-(b)

$E q .(A)=2 \int d \rho \int d \phi \int_{0}^{\infty} d z \rho z \Psi_{i}(\rho, \phi, z) \cos (k z)=$ non - zero strong intensity

Case (2): BTA adsorbed flat on $\mathrm{Cu}$ and the light's polarization is vertical to the surface, as shown in Figure 5-(B)-(c)

$E q .(A)=-2 i \int d \rho \int d \phi \int_{0}^{\infty} d z \rho^{2} \cos (\phi) \sin (k z) \Psi_{i}(\rho, \phi, z)=$ non - zero strong intensity

Case (3): BTA stands up, facing the light's propagation and the light's polarization is vertical to the surface, as shown in Figure 5-(B)-(d)

$E q .(A)=\int d \rho \int d z \int_{0}^{\pi} d \phi \rho z e^{-i k z} \Psi_{i}(\rho, \phi, z)+\int d \rho \int d z \int_{-\pi}^{0} d \phi \rho z e^{-i k z} \Psi_{i}(\rho, \phi, z)=0$

, due to the anti-symmetric property of $\Psi_{i}(\rho, \phi, z)$, the global $\pi$-molecular orbital P3.

Case (4): BTA stands up, facing the light's propagation and the light's polarization is parallel with the surface, as shown in Figure 5-(B)-(e)

$E q .(A)=\int d \rho \int d z \int_{0}^{\pi} d \phi \rho^{2} \cos (\phi) e^{-i k z} \Psi_{i}(\rho, \phi, z)+\int d \rho \int d z \int_{-\pi}^{0} d \phi \rho^{2} \cos (\phi) e^{-i k z} \Psi_{i}(\rho, \phi, z)=0$, due to the anti-symmetric property of $\Psi_{i}(\rho, \phi, z)$, the global $\pi$-molecular orbital P3.

By summarizing the above four cases when BTA adsorbs flat on $\mathrm{Cu}$ surface, UPS intensity of P3 is strong with nonzero term in equation (A) regardless of polarization direction such as non-polarized UV source. On the other hand, when BTA stands up on $\mathrm{Cu}$ surface facing the light propagation, the UPS intensity is very weak with zero term in equation (A). This kind of symmetry argument is very useful to determine the molecular orientation and can be expanded to NEXAFS and XANES as well as UPS.

Our model explains the behavior of P3's UPS intensity in Figure 5-(a). Therefore, we conclude that BTA adsorbs flat on $\mathrm{Cu}$ surface in the beginning, but vertical BTA molecules appear at higher concentration and form polymerization. However, we could not determine whether the vertical polymerization occurs at one monolayer with higher coverage or it occurs at the $2^{\text {nd }}$ monolayer and above. Another separate Scanning Tunneling Microscope (STM) measurement ${ }^{\text {xii }}$ was made by Dr. Keyhyun Cho, as shown in Figure 6. STM image confirms flat adsorbed BTA and vertically oriented polymerized BTA's chain. Therefore, we suggest a new polymerization model that BTA makes flat absorption like shields on $\mathrm{Cu}$ surface first, and then makes vertically oriented polymerization like a chain that binds shields or fills space between shields to protect $\mathrm{Cu}$ surface. This property is very important and useful for a fabrication of a micro-chip that can connect organic polymers and bio materials because BTA can grab the $\mathrm{Cu}$ atoms on the surface with strong $\pi-$ bond and capture external organic polymers with their polymer chains.

\section{Summary}

We measured thickness dependent UPS data of BTA on $\mathrm{Cu}(110)$ surface. Our theoretical model based on the symmetry property of P3 molecular orbital indicates that BTA changed molecular orientation during deposition. The developed symmetry calculation method is very general and can be also expanded to NEXAFS and XANES. The measured 
polymerization process of BTA shows that BTA is a good candidate material that can anchor external organic molecules on $\mathrm{Cu}$ electrodes in microelectronics fabrication process.

\section{Acknowledgement}

This presentation was supported by Creativity and Innovation Program of NASA Langley Research Center. The authors appreciate Dr. Jaewoo Kim at STC, Hampton, VA for AFM data and Dr. Keyhyun Cho at Yeungnam University, South Korea who studied BTA molecules with STM and assisted us.

\section{Figures}
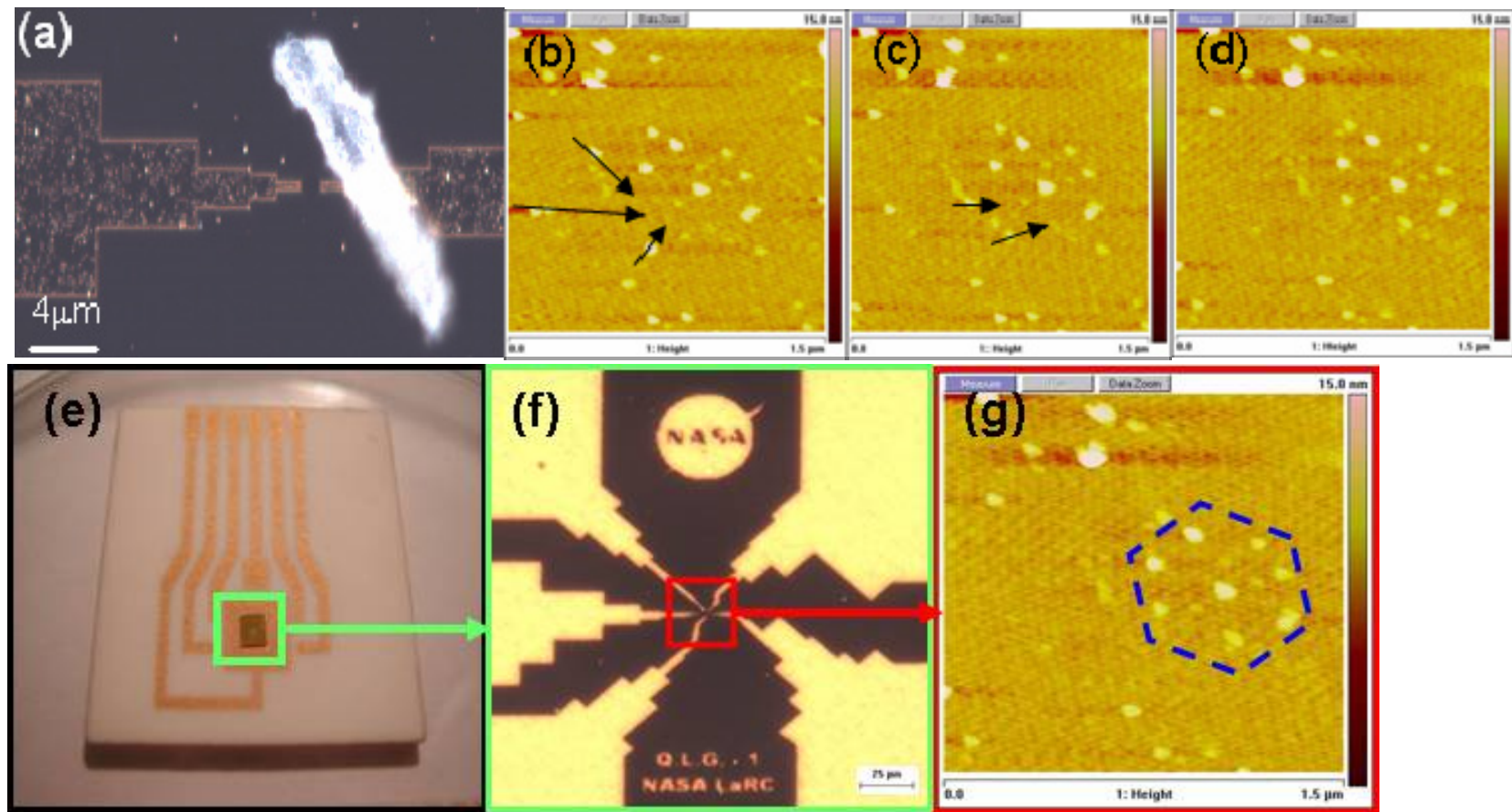

Figure 1 - (a) Interconnection failure of polymer structure to Au electrodes, (b) $\sim$ (d) Manipulation of bio-protein , Ferritins with AFM tip: the arrow shows the motion of Ferritins as a result of AFM tip manipulation, (e) microchip in a few $\mathrm{cm}$ size, (f) metal electrode area in the microchip, (g) arranged Ferritin proteins in the center of electrode junction point. The interconnections to these proteins are still missing.

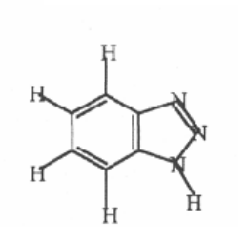

$$
\text { Benzo Triazole(BTA) }
$$
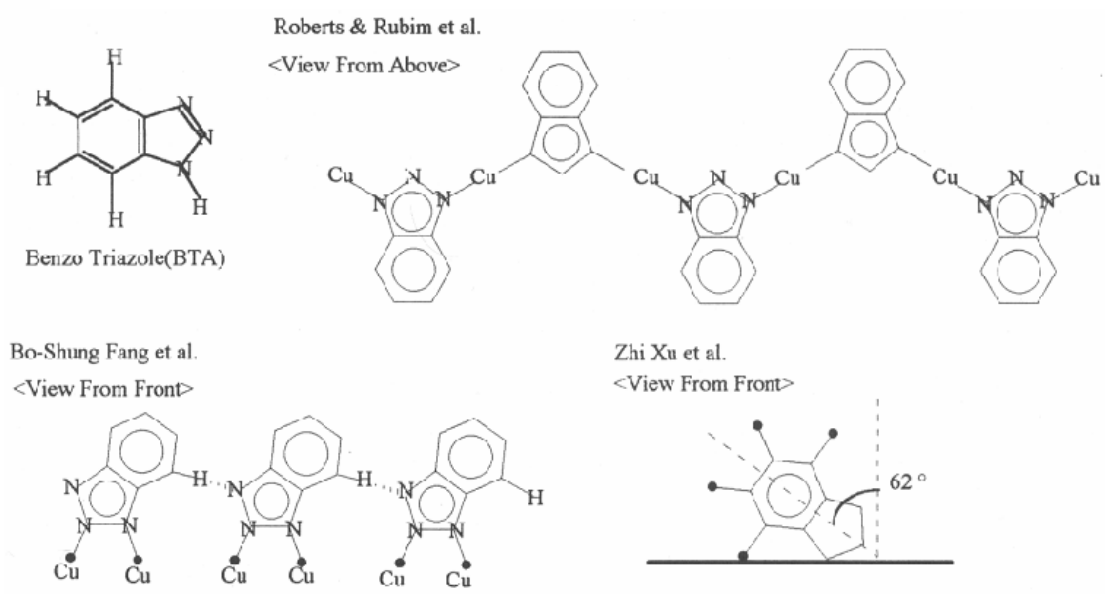

\section{Zhi Xu et al.}

$<$ View From Front>

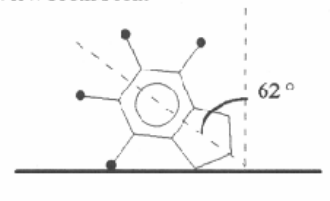

Figure 2 - BTA molecular structure and previously suggested adsorption models on Cu surface 

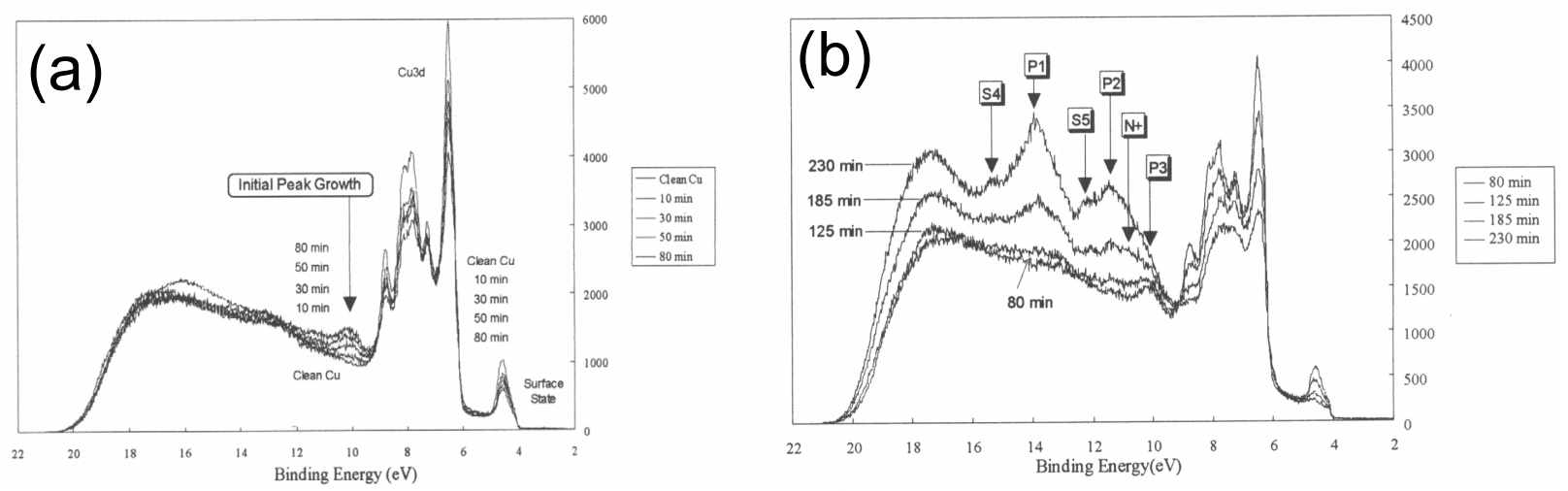

Figure 3 - (a) UPS data of BTA deposit up to 80 min., (b) UPS data of BTA deposit from 80 min. to 230 min.
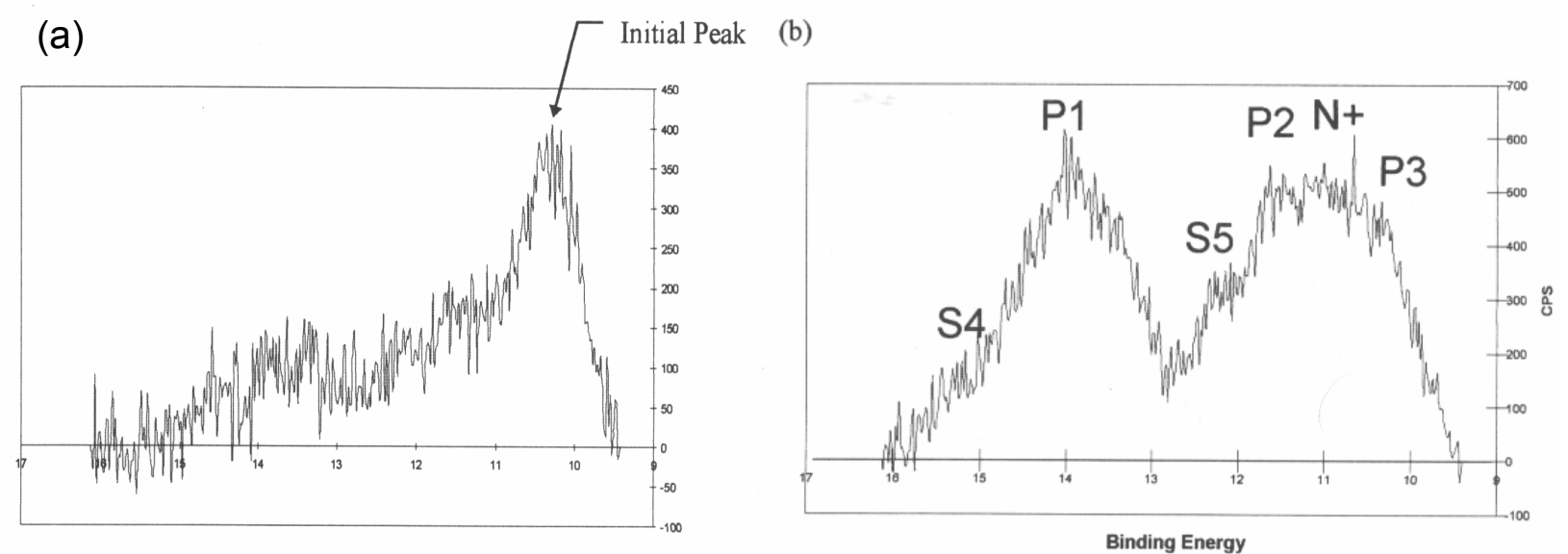

Figure 4 - Comparison of UPS data of BTA deposit between (a) 80 min. and (b) $230 \mathrm{~min}$. 


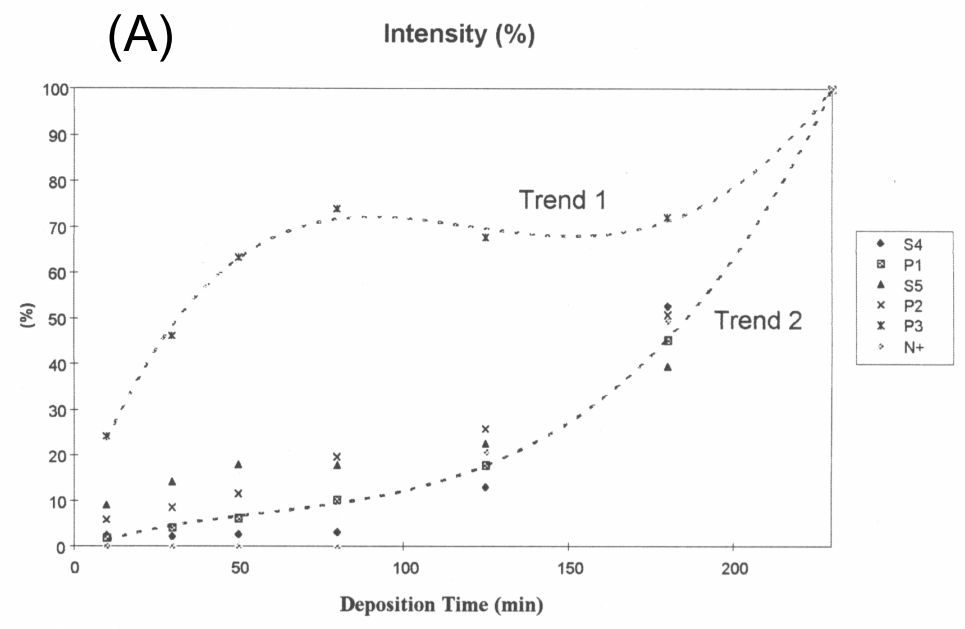

(a) Antisymmetric Property of BTA $\pi$ MO $\quad \Psi_{(\mathrm{z})}=-\Psi_{(-\mathrm{z})}$ for (b), (c)

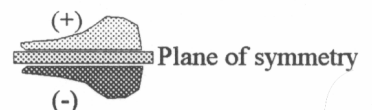

$\Psi_{(\varphi)}=-\Psi_{(-\varphi)}$ for $(\mathrm{d}),(\mathrm{e})$

(B)

(b)

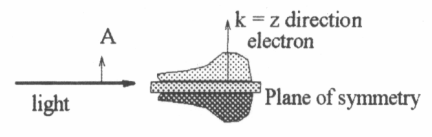

(c)

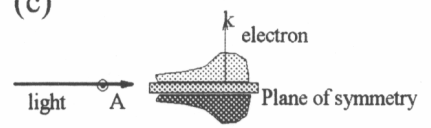

(d)
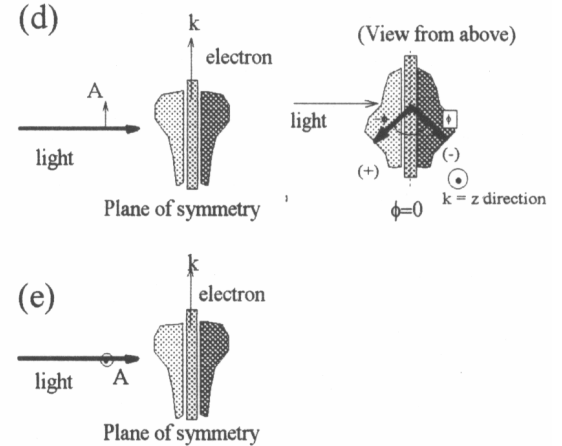

Figure 5 - (a) Relative Intensity Change and (b) Orientation of UV Polarization and BTA Molecules

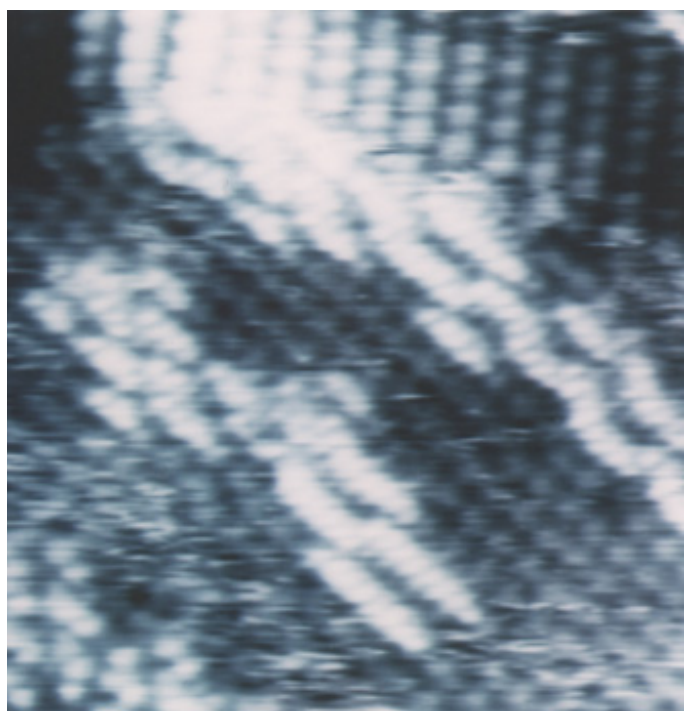

Figure 6 - STM Image of BTA layers on Cu surface, Courtesy of K. Cho and T. Sakurai ${ }^{\mathrm{xi}}$ 


\section{Reference}

${ }^{\mathrm{i}}$ G.D. Watt, R.B. Frankel, G.C. Papaefthymiou, PROCEEDINGS OF THE NATIONAL ACADEMY OF SCIENCES OF THE UNITED STATES OF AMERICA 82 (11), 3640-3643 (1985)

${ }^{\text {ii }}$ R.K. Watt, R.B. Frankel, G.D. Watt, Biochemistry 31 (40), 9673-9679 (1992)

${ }^{\text {iii }}$ R.C. Jaeger, Introduction to microelectronic fabrication, Modular Series on Solid State Devices Vol. 5, ${ }^{\text {nd }}$ Ed., Prentice Hall, (2002)

${ }^{\text {iv }}$ A.A. Istratov, C. Flink, H. Hieslmair, E.R. Weber, T. Heiser, Phys. Rev. Lett. 81, 1243-1246 (1998)

${ }^{v}$ P. C. Andricacos, C. Uzoh, J. O. Dukovic, J. Horkans, and H. Deligianni, IBM Journal of Research and Development, 42 (5), http://www.research.ibm.com/journal/rd/425/andricacos.html (1998)

${ }^{\text {vi }}$ Y. Park, H. Noh, Y. Kuk, K. Cho, T. Sakurai, Journal of Korean Physical Society 29 (6) pp.745-749 (1996)

${ }^{v i i}$ R.F. Roberts, Journal of Electron Spectroscopy and Related Phenomena 4, p.273 (1974)

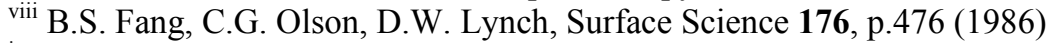

${ }^{\text {ix }}$ Z. Xu, S. Lau, P.W. Bohn, Langmuir 9, p.993 (1993)

x J.F. Walsh, H.S. Dhariwal, A. Gutierrez-Sosa, P. Finetti, C.A. Muryn, N.B. Brookes, R.J. Oldman, G. Thornton, Surface Science 415, p.423 (1998)

${ }^{x i}$ M.H. Palmer and S.M.F. Kennedy, Journal of Molecular Structure 43, p.203 (1978)

xii K. Cho, J. Kishimoto, T.Hashizume, T. Sakurai, Japanese Journal of Applied Physics Part 2-Letters 33 (1b) pp.1125$1128(1994)$ 